\title{
Las mujeres españolas en la Resistencia francesa
}

\author{
M. ${ }^{\text {a Fernanda ManCebo* }}$ \\ (Universidad de Valencia)
}

La aproximación metodológica que constituye el objeto de este estudio comprende dos aspectos. En primer lugar, un estado de la cuestión respecto a bibliografía y fuentes, tanto más necesario cuanto el tema de la participación de los españoles en la "resistencia» francesa, no fue tratado en el Coloquio de Salamanca '. Y en segundo término, un "estado de la cuestión" referido al conocimiento que hoy tenemos sobre la participación de las mujeres. En él se trata de sistematizar en algunos epigrafes, la información que sobre la misma proporcionan la bibliografía y fuentes de archivo y hemerográficas, además de testimonios orales en su mayoria ya publicados. Después de varios esfuerzos puedo utilizar el testimonio de Juan Marín Garcia, que alude en su escrito al papel desempeñado por las mujeres, compañeras de ciertos líderes de la resistencia. $Y$ enumerar algunas publicaciones recientes, aunque la mayor parte no centradas en Francia, sobre mujeres en el exilio ?

Pero, sin duda, es fundamental tanto en uno como en otro apartado la obra y consejo de Neus Catalá, ella misma resistente y deportada.

* Dedicado a Carmen Caamaño, resistente en el interior.

Reseña de los Coloquios celebrados en Salamanca (mayo 1991) y Turin (junio 1991). Bulletin trimestriel IHTP, $\mathrm{n}^{\circ} 44$, París, juin 1991, p. 6. Una primera versión de este escrito se presentó en el Congreso Internacional de Paris en 1991.

2 P. Dominguez: Voces del exilio. Mujeres españolas en México, 1939-1950, Madrid, 1994. VV.AA. Nuevas Raices. Testimonios de mujeres españolas en el exilio. G. Medrano (coord.), Valencia, 1995. VV.AA. Homenaje a Manuela Ballester, M. GARcia (coord.). Valencia, 1995. VV.AA. Exiliados. Obra y memoria del exilio valenciano en América, M. a F. MANCEBo; A. GiRona (Coord.), Valencia, 1995. Exiliados, M. Garcia (coord.), 3 vols., Valencia, 1995. Para Francia, R. Duroux, "Historia y desmemoria. Prácticas culturales en los refugios de mujeres españolas en Francia, 1939-1940", en Mélanges Louis Cardaillac, Zaghouan, FTERSI, abril de 1995. Tambièn me ha resultado muy valiosa la obra e información oral de Eduardo Pons Prades. 
En el trabajo se prescinde de las memorias y actuación de personalidades significativas y más conocidas como Victoria Kent, Federica Montseny, Isabel de Palencia, María Casares, Teresa Pàmies, Margarita Nelken o Constancia de la Mora, entre otras. Su importancia está fuera de toda duda, pero en este estudio quisiera atender a esta heroína colectiva, "la mujer española», cuyo nombre y apellidos puede aparecer en algún caso, pero en otros ni se ha podido recoger. Precisamente Neus Catalá ha constatado en 25 de los 90 departamentos franceses la existencia de mujeres resistentes luego desaparecidas. Más de 400 mujeres de las que no queda huella ni recuerdo ${ }^{3}$. También el poeta asturiano, José M.. Álvarez Posada, "Celso Amieva» en 1944, reconoce:

Creo que deberíamos rendir un cálido homenaje a "nuestras mujeres" de las que con frecuencia nos hemos olvidado. Sin ellas, bien lo sabes, nosotros "los valientes", "los heroicos" guerrilleros, nos hubiéramos hundido moralmente más de una vez... ${ }^{4}$.

Por ello envía a Eduardo Pons un poema titulado «Muchachas del maquis" que comienza "Quiero nombrar aquí a las compañeras/abnegadas y anónimas, enlaces/y escuchas, auxiliares guerrilleras/o heróicas enfermeras...». Nombra a unas cuantas pero muchas más desaparecieron sin nombre. Los que conocieron los hechos dicen que no hay que hablar de cientos sino de miles.

\section{BIBLIOGRAFIA Y FUENTES}

En el inicio del proyecto de los tres coloquios que culminó con el de París, el Boletin del IHTP ${ }^{5}$ publicó un utilísimo y necesario estado bibliográfico de la cuestión.

Ateniéndome al realizado para los españoles por Geneviève DreyfusArmand, se observa, en primer lugar, la exigua cantidad de títulos -87 por todos los conceptos- frente a los 317 de la bibliografía recogida por Eric Vial para los italianos.

3 Conversación mantenida el 26-VIII-1991. N. CATALA, De la resistencia y la deportación. 50 testimonios de mujeres españolas. Barcelona, 1984.

4 Recogido en E. PONS PRADEs, Republicanos españoles en la 2. guerra mundial. Barcelona, 1975, p. 22.

5 Bulletin trimestriel IHTP, $\mathrm{n}^{\circ} 40$, París, juin 1990. Este coloquio internacional está publicado con el titulo Exils et migration. Italiens et Espagnols en France: 1938-1946. Sous la direction de Pierre Milza et Denis Peschanski, Editions I'Harmattan, París, 1994. 
Esta relación, casi exhaustiva ${ }^{6}$, autoriza el acuerdo con Antonio Vilanova y con el título que da a uno de los primeros estudios sobre el exilio español en Francia, Los olvidados. Los exiliados españoles en la segunda guerra mundial (1969). Olvido que también reconoce L. Stein con estas palabras: «la historiografía de la Resistencia y liberación francesa había olvidado, en gran parte, las contribuciones hechas por los republicanos españoles a la victoria final»? .

Pero hoy, más de veinte años después del libro de Vilanova, ¿cuál es el panorama? La historiografía francesa y también, y fundamentalmente, la española, sigue sin prestar demasiada atención al tema, exceptuando estos últimos coloquios, aunque desde 1991 se ha intensificado significativamente el trabajo en este ámbito.

Pero, ¿qué decir de los estudios sobre la contribución de las mujeres? Cuando por sugerencia de uno de los españoles resistentes, el citado Juan Marín (19 años en 1939) me propuse abordar este estudio, empezaron las dificultades. ¿Bibliografía? ¿Fuentes de archivo, impresas, orales? Un gran vacio empezó a configurarse en torno ${ }^{8}$.

Pero en tanto que inexplorado, más despertaba nuestro interés. En todo caso, llegué a la conclusión de que poco más puede hacerse por el momento, que un específico estado de la cuestión. Las mujeres, más aún, eran las grandes olvidadas. Bibliografía y las fuentes para el estudio de su participación se presentan como el problema más complejo a resolver. Aura Roces en 1946, poco después de la liberación de Francia, habia escrito:

En esta hora de entusiasmo, como en otras de más libertad, encontramos la triste ausencia de un sector: el femenino; nadie o muy pocos se ocupan de las mujeres y nosotras indiferentes a ese aislamiento en que se nos deja nada hacemos por aparecer en el escenario social como factor capital para la obra de la redención y emancipación de los pueblos... ${ }^{9}$.

¿Qué encontramos sobre las mujeres en el estudio bibliográfico anteriormente aludido?

6 Completada por JAvier RuBio, "Coloquio "Españoles en Francia, 1936-1946", consideraciones bibliográficas previas", Studia Historica. vol. VIIl, Salamanca, 1990, pp. 187-08; y después por Geneviève Dreyfus en noviembre del mismo año.

' L. StEIN, Más alla de la muerte y del exilio, Barcelona, 1983, p. 321.

a Vacio que se continúa hoy, al menos para la situación en Francia.

9 A. ROCES, "Manantial de Vida y Libertad", La voz del exilro, Boletín de información. Año I, $n^{\circ} 1$ del MCE-CNT en Francia. Editado por la regional de Dijon, enero de 1946. 
En el apartado de Memorias y testimonios generales, están incluidas las obras clásicas de Federica Montseny, Isabel de Palencia y Teresa Pàmies. Sobre Guerra y resistencia de nuevo el testimonio de Federica Montseny, Cien días de la vida de una mujer. Y la intervención de Neus Catalá en el Coloquio Les femmes dans la resistance, "Dans une lutte ininterrompue contre le fascisme, participation des femmes espagnoles à la Resistence française" (1975). Finalmente, aunque no aparece en esta bibliografía, se cuenta con el artículo, resumen de un DEA (1984) de MarieJosé Nadal, "Femmes de l'exil espagnol» ${ }^{10}$. Estudio que, basado en el testimonio de tres mujeres exiliadas, se refiere fundamentalmente a la evolución y formación ideológica y política de la mujer española hasta 1939.

Entre bibliografia y fuentes -en ese espacio que es difícil diferenciar en este tipo de trabajos y temas - se encuentran las obras de Miguel Ángel Sanz, Eduardo Pons Prades o Antonio Soriano.

Si se atiende a la tradicional clasificación de las fuentes. De archivo, hemerográficas u orales, o bien públicas y privadas, encontramos igual dificultad.

Los archivos de la policía o judiciales franceses cuando se refiere a procesos personales no se pueden consultar hasta pasados 100 años de los hechos ${ }^{11}$. Los archivos del PC, PSOE (Fundación Pablo Iglesias, Fundación Largo Caballero) o del movimiento libertario en Amsterdan o en el Histórico Nacional de Salamanca guardan una documentación muy rica pero fragmentaria en lo que a Mujeres se refiere ${ }^{12}$. La comisión española de la FNDIRP, ya disuelta, no tiene archivos ${ }^{13}$. La labor hemero-

10 M. JOSE NADAL, "Femmes de l'exil espagnol. Formation idéologique et action politique des femmes du peuple ayant choisi de s'exiler en France en 1939", Materiaux pour l'histoire de nostre temps, BDIC, № 15 , janv-juin 1989, 28-37.

"Sobre los archivos franceses, sus restricciones y posibilidades D. PESCHANSKI, "Sources françaises pour une histoire des espagnols en France, 1938-1946», Españoles en Francia, 19361946, Salamanca, mayo 1991, pp. 97-106.

12 Los fondos del archivo del Partido Comunista son, sin duda, indispensables para clialquier trabajo en relación con el exilio en Francia. La sección "Equipo de Pasos" proporciona intormación sobre la actividad de las mujeres en el conjunto de la acción guerrillera. La sección "Unión de Mujeres Españolas" se refiere principalmente a la actividad de esta organización a partir del verano de 1946 cuando se celebra el primer congreso. Las publicaciones consultadas se refieren básicamente al interior como veremos a continuación. JoSÉ A. DE MINGO, V. RAMOS, "Fuentes para el estudio del exilio español en Francia (1936-1946): El archivo del comité central del partido comunista de España", Españoles en Francia, 1936-1946, pp. 47-64. En la misma publicación puede consultarse las fuentes de la Fundación Pablo Iglesias; Centro de Estudios Históricos Internacional (CEHI), a cargo de Joan Oliver y María Lourdes Prades; y otros de menor importancia para nuestro objeto.

13 Las gestiones realizadas para localizar algunas publicaciones han sido infructuosas por ejemplo La voz de los olvidados, Bulletin d'information de l'Association d'anciens combattans et vic- 
gráfica realizada proporciona datos muy escasos para el papel de la mujer en la resistencia.

Generalmente publicaciones como Reconquista de España, Nuestro combate, e incluso la prensa especifica de mujeres como Mujeres antifascistas españolas o Companya, hacen más referencia a la España interior, a la terrible represión franquista de los años 40 y 50 que a los acontecimientos del exilio ${ }^{14}$. Como ejemplo hay alusiones a Mujeres en La Voz de España, $n^{\circ} 2$ (27-X-1944) y no 17 (16-II-1945); o Reconquista de España, no 39 (Toulouse, septiembre 1944) y no 50 (París, 13-l-1945). Algunos folletos de la Unió de Dones de Catalunya, se dirigen a su reorganización.

Finalmente el libro de Antonio Soriano ${ }^{15}$ aunque contiene 17 testimonios, sólo uno es de una mujer, la modista Rosa Laviña.

Por otra parte, la profesora Olga Querol, de la Universidad de Toulouse ha elaborado en soporte de imagen el testimonio de varias mujeres del sur de Francia, de las cuales sólo una participó en la resistencia, Visitación Maigi (Toulouse) ${ }^{16}$.

Restan los testimonios orales aunque he fracasado en cualquier intento de conseguir alguno nuevo de mujer. Los más importantes están publicados en el sobrecogedor libro de Neus Catalá ya mencionado. En él, después de improbo trabajo, publica las entrevistas de más de 50 mujeres. Asimismo los dos libros de Miguel Ángel Sanz, quizá la historia más completa de la resistencia española recoge nombres y actuación de algunas mujeres ${ }^{17}$. El libro de Eduardo Pons -él mismo también protagonistaRepublicanos españoles en la $2^{a}$ guerra mundial y la serie "Memorias del exilio", del mismo autor, proporciona bastantes noticias ${ }^{18}$.

times de guerre de la Republique Espagnola. La Mairie du Boulou, donde se ubica la redacción no contesto, ni tampoco el redactor-jefe Luis Romero. En cambio, Luis Garcia Manzano, uno de los colaboradores nos proporciono algunas direcciones que nos han sido de suma utilidad, y la información sobre la FNDIRP mencionada en el texto.

14 Agradezco a Jordi Planes la información sobre las existencias en el CEHI. El periódico Companya, órgano del Consell nacional de la Unió de Dones de Catalunya, aunque editada en el exterior (Toulouse), está fundamentalmente dirigida al interior "La Unió de Dones de Catalunya, no és una organització de l'exili. Es una organització creada a Catalunya". Es por ello que su contenido se dirige a la España sometida al franquismo. Intervención de TERESA SERRA, Companya, nº 2 Extraordinari (Sept.-oct.) 1946, p. 2.

15 A. Soriano, Exodos. Historia oral del exilio republicano en Francia. 1939-1945. Barcelona, 1989. Recientemente contamos con el audiovisual A.A.V.V. Exilios. Refugiados españoles en el mediodia de Francia, UNED, Madrid, 1994, en el que aparecen diversos testimonios de mujeres.

16 Debo esta información a Olga Querol (16-VIH-1991).

17 Miguel Ángel Sanz, Los guerrilleros españoles en Francia, La Habana, 1971; y l.uchando en tierras de Francia, Madrid, 1981.

18 E. PONS Prades, "Memorias del exilio", publicadas en los números 12-13-14-15-16 y 17 de Historia 16, abril-septiembre 1977. Sus libros están recogidos en la Bibliografia de Geneviève Dreyfus. 
Esto es con lo que, por el momento, contamos sobre mujeres en la resistencia, a salvo siempre cualquier error, omisión o ulteriores investigaciones.

\section{LA MUJER ESPAÑOLA DE LA «RESISTENCIA»}

Con estos escasos elementos y utilizando el contexto general, trataré de esbozar un perfil de la joven o mujer resistente «anónima». Aquélla que, pese a su escasa formación político-intelectual, en ocasiones fue capaz de guardar los secretos bajo las torturas, fue deportada y desapareció en los temibles campos de exterminio, especialmente Ravensbrück. O bien afrontó las adversidades y logró sobrevivir, volviendo incluso a España para proseguir la lucha clandestina hasta el derrocamiento del franquismo.

Como dice Jean Cassou «La guerra civil española no se terminó con la victoria de Franco... Los exiliados en Francia siguieron luchando en la batalla de Francia y en las diversas formas de participación española en la Resistencia francesa". Y esta es la primera connotación de la «resistencia" de los españoles y franceses. Ellos tenían la convicción de que proseguian una lucha por la democracia y contra el fascismo que había comenzado en España. Esto explica en mucha parte su participación, a pesar de la cruel acogida que recibieron al cruzar la frontera, en los campos de refugiados del sur.

Otra nota previa es la organización y estructuración de la resistencia española, desde los primeros tiempos «un movimiento español de resistencia que llegó a formar unidades de guerrilleros que combatieron hasta la liberación en la mayoría de los departamentos» ${ }^{19}$.

Entre agosto de 1941 y septiembre de 1942 el Partido Comunista creó el movimiento de resistencia «Unión Nacional Española» que aglutinó a los españoles, especialmente comunistas que ya trabajaban en este sentido desde el comienzo de la guerra. La UNE incluyó también elementos políticos dispares, monárquicos, tradicionalistas navarros y CEDA ${ }^{20}$ y tuvo su sección de Mujeres. Por su parte, los anarquistas crearon la Alianza Democrática Española que agrupó a la izquierda no comunista y en la que actuaron también numerosas mujeres.

19 M. ÁnGel, Luchando en tierras..., p. 41.

20 L. Stein, Más allá de la muerte..., p. 172. 
Posiblemente la participación de los españoles se hubiera dado igual $\sin$ estas organizaciones, pero sin duda no hubiera sido tan efectiva ${ }^{21}$.

\section{Origen geográfico. Edad. Estado civil. Niños}

Las resistentes proceden de toda la geografía española. Pero la caída de la zona noreste dio lugar a una mayor presencia de catalanas, aragonesas, navarras y vascas. Valencianas y madrileñas pasaron la frontera ya con la condición de refugiadas, especialmente las segundas. Los republicanos de Murcia, Andalucia, las Castillas, Asturias, León y Galicia, bien porque formaban la llamada «zona nacional» o por haber resistido hasta el final, llenan las cárceles o los cementerios o salen, los que pueden hacia el norte de África.

Casi todos los testimonios señalan que pasaron la frontera entre enero y febrero de 1939, algunos indican incluso "al final de nuestra guerra». Es decir que para la mayoria la caída de Cataluña representó el final; aunque haya que señalar el compromiso de los combatientes que regresaron a defender la zona centro.

Un pequeño porcentaje residía ya en Francia cuando comenzó la guerra, integrando la llamada «emigración económica». Según testimonio de Jesusa Bermejo "muchos españoles residentes como nosotros marcharon a defender a la República... las mujeres organizamos la ayuda y nos adherimos a una organización que se dedicaba a esta ayuda. Nosotras empezamos nuestra resistencia contra el fascismo en 1936" ${ }^{22}$. Alguna incluso procedía de familia residente en Francia desde principio de siglo (1904). Esta mujer cuando empezó a trabajar en la resistencia tenía veintidós años. Pasó por Ravensbrück. Logró volver a su casa a Perpignan después de la liberación. Más aún, reencontró a su marido, refugiado español, que habria sido internado en Dachau. Un acontecimiento casi increible ${ }^{23}$.

Maria González, asturiana, había llegado a Francia en 1923. Su padre y hermano habían quedado «inútil total» en las minas de Asturias. También de esta tamilia tres miembros volvieron voluntarios para luchar en España ${ }^{24}$.

\footnotetext{
M. Ángel, Los guerrilleros españoles..., p. 35.

N. Catalá, De la resistencia..., Jesusa Bermejo, p. 69.

3 N. Catala, De la resistencia... Mercedes Bernal, p. 73.

N. Catala, De la resistencia..., p. 151.
} 
Carmen Asensi llegó a Nîmes antes de la guerra del 14. El caso de esta valenciana es de los más impresionantes.

Respecto a la edad. El denominador común es la juventud. En muchos casos plena adolescencia. Casi todas dicen que "tenian conciencia de los peligros que corrían". "Lo que yo he vivido, lo que yo he sufrido, yo me lo he ganado". Asi comienza el testimonio de Neus Catalá, nacida en 1914.

Las más jóvenes de las entrevistadas tenían 15-16 años. Josefa Bas empezó a trabajar con el «maquis de Dordogne» a los 16 años. Lina Bosque actuaba de enlace o acompañaba a los hombres que decían pasaban más desapercibidos con ella:

Me acuerdo que cerca de donde estábamos nosotros (Roche-Segur) nabía un campamento de alemanes y siempre había centinelas... Me echaban piropos (yo era joven, como digo) pero yo jestaba tan nerviosa!... ${ }^{25}$.

Carmen Torres, 15 años, cuando pasó a Francia. Alfonsina Bueno tenía 17 años cuando empezó la guerra. Cuatro años después, con 21 años, mientras su marido estaba internado en el campo de castigo de Vernet ella empezó a trabajar para el «maquis» con el maestro valenciano Paco Ponzan Vidal. Su hija (¿6-7 años?) «con disimulo, indicaba el camino de la estación a los que pasaban los Pirineos". El día de su detención (1943) su hija Angelina tenía 9 años.

Pilar Claver, navarra, huida hacia Barcelona a través de los Pirineos y después de allí a Francia, llegó con 20 años al "campo" de Angôuleme. Allí conoció a Margarita y María, chicas catalanas de las Juventudes del PSUC. Ella, personalmente no se sentía tan joven. "Nuestra guerra, tan cruel, me había madurado". Antonia Frexedes, internada primero en el campo de Agde, después en el de castigo llamado de Noé y finalmente deportada a Ravensbrück, tenía 23 años cuando empezó la guerra de España.

En fin, era lógica esta presencia joven en la «resistencia» y también por ello nos ha podido llegar su testimonio. Mujeres de 40, 50 años no pudieron resistir el trabajo, las privaciones y los sufrimientos. No han podido llegar hasta nosotros.

Y como se ha visto en los ejemplos señalados era bastante frecuente la joven-mujer casada o unida a algún compañero, igualmente guerrillero o resistente, antiguo combatiente en la guerra de España, después integrado en el "maquis".

2. N. Catala, De la resistencia..., Lina bosque, p. 75. 
La suerte de los hijos iba unida a la de estas mujeres. Niños de meses o de escasos años, a los que la solidaridad de las compañeras salvó de perecer en algún caso, aunque lo habitual era la muerte. En el campo de Saint Cyprien «El agua salía podrida, y tanto los niños como los adultos tenían colitis. Los niños se morían como moscas, pues las madres estaban obligadas a hacer los biberones con esa podredumbre... ${ }^{26}$.

Más tarde, en casa de esta mujer, Filomena Folch, se escribia el periódico "Reconquista de España».

Filo Formes, viuda de Solé, escribe en su testimonio: "Yo tenía conmigo a mis dos hijas de catorce y ocho años de edad... El enlace con París y otras ciudades lo realizaba mi hija que entonces contaba con diecisiete años" (1943).

La suerte de los "niños» del exilio es una "asignatura pendiente como dicen Rose Duroux y Raquel Thiercelin ${ }^{27}$. Pero indudablemente participaron ellos también, como sus madres, en la resistencia ${ }^{28}$.

\section{Extracción social}

Como primera aproximación y a falta de cuestionarios especialmente referidos a este punto, creo se puede aventurar una hipótesis: La mujer que participa en este aspecto de la "resistencia", las guerrillas, la lucha armada, no procede de la burguesia. Proviene básicamente de las capas populares rurales o urbanas de la sociedad española. Es una joven o mujer "del pueblo» que ha sido conmocionada por los acelerados cambios socioeconómicos que se producen en España en las décadas veinte y treinta.

Diversos estudios se han centrado en esta toma de conciencia de la mujer trabajadora o incluso hija o ama de casa. La intensificación de la

26 N. Catala, De la resistencia..., Filomena Folch, p. 133.

27 R. DuRoux y R. THIERCElin, “Los niños del exilio: Asignatura pendiente", Españoles en Francia, 1936-1940, Salamanca, 1991, pp. 437-450. Recientemente el catálogo de la exposición El exilio español en la guerra civil: los niños de la guerra, con texto de A. Alted, Archivo Histórico Nacional, sección Guerra Civil, Ministerio de Cultura y Fundación Largo Caballero, Madrid, 1995. Es de gran utilidad la bibliografia mencionada en el catálogo, especialmente $P$. Marqués, Les enfants espagnols réfugiés en France (1936-1939), AutoEdition, Paris, 1993. E. Zafra, R. Crego y C. Heredia, Los niños españoles evacuados a la URSS en 1937, Madrid, 1989

28 JESUSA BERMEJO continúa: "Una hermana mía, desesperada de no tener nada más que dar a sus hijos (tenia cinco hijos, la menor de seis meses), y teniendo que sufrir la carga de un marido alcohólico, se suicidó tirándose al Sena... ¡Cinco bocas más! 
lucha obrera, la consolidación de los partidos políticos obreros con sus juventudes. La proclamación de la República - con la concesión del voto-, la revolución de octubre de 1934, el triunfo del Frente Popular, y, especialmente, la guerra civil son hechos que afectan su status socio-económico y hacen tomar conciencia de sus derechos y deberes políticos a la mujer española.

Tres núcleos de testimonios avalan esta hipótesis. Los libros de Giuliana Di Febo y Mary Nash ${ }^{29}$. Los testimonios de los impresionantes libros de Tomasa Cuevas ${ }^{30}$ y Consuelo Garcia ${ }^{31}$ sobre la represión en el interior; el artículo de Marie-José Nadal y finalmente el ya varias veces citado de Neus Catalá. La imagen de la mujer, antifascista y demócrata ante todo, que sugiere esta literatura está más cerca de la mujer del pueblo que de la intelectual o republicano-burguesa educada en la Institución Libre de Enseñanza, que también sin duda participó. Para decirlo de una vez y utilizando los testimonios de Marie-José Nadal:

N'étant pratiquement pas allées à l'école, elles ont dû travailler très jeunes (entre 8 et 14 ans) dans l'industrie textil et ont milité dans les syndicats ${ }^{32}$.

O la descripción que de su familia hace Tomasa Cuevas: "Mi abuelo materno era albañil; el paterno hornero. Mi padre era un niño cuando empezó a trabajar en una fábrica de harina del pueblo... Mi madre trabajaba todo lo que la pobre podia; por las mañanas cocía pan para gente del pueblo... después lavaba ropa en el lavadero municipal..." ${ }^{33}$. Y la misma Neus Catalá «Hija de campesinos, y ella misma campesina hasta que estalla la guerra civil». Otras dan constancia de trabajadora industrial u obrera calificada.

Esta es la joven mujer ejemplar que consagrará lo mejor de su vida a la lucha contra la dictadura franquista en el interior o contra el fascismo-nazismo en la "resistencia» francesa ${ }^{34}$.

2s MARY NASH, Mujer y movimiento obrero en España, 1931-1939, Barcelona, 1981. G. DI FEBO, Resistencia y movimiento de mujeres en España (1936-1976), Barcelona, 1979.

30 Tomasa Cuevas, Mujeres de las cárceles franquistas, 2 vols.: I, Madrid, s/a; II. Barcelona, 1985.

31. Consuelo Garcia, Las cárceles de Soledad Real, Madrid, 1987.

32 Marie-José NADAL, «Femmes de l'exil espagnol", Materiaux no 15, p. 28.

3.3 T. Cuevas, Mujeres de las cárceles tranquistas..., p. 17.

34 Para la guerrilla interior F. Romeu, La Agrupación Guerrillera de Levante, Valencia, 1987, Las mujeres, pp. 120-ss. Y C. ALCALDE, La mujer en la guerra civil española, Madrid, 1976. Y de la misma autora, Mujeres en el franquismo. Exiliadas nacionalistas y opositoras, Madrid, 1996. F. ROMEU, El silencio roto... Mujeres contra el franquismo, autoedición, Asturias, 1994. 


\section{Opción político-ideológica}

De lo apuntado en el apartado anterior ya se deduce que la conciencia de clase a la que han accedido duramente durante su niñez o juventud va a unificar dentro de la diversidad la opción político-ideológica de la mujer del pueblo exiliada.

Para empezar, la mayoría de ellas han escogido, o se han visto abocadas al exilio y a la resistencia precisamente por su implicación política. Lo cual no quiere decir que en la gran desbandada hacia la frontera, tras la caída de Cataluña, no hubiera muchísimas mujeres campesinas o de las ciudades. Y sin filiación política expresa, fueron arrastradas por el pánico general ante los bombardeos y la anunciada llegada de las tropas enemigas.

No todos los testimonios recogidos por Neus Catalá explicitan la pertenencia o militancia en partidos políticos. Pero creo se puede afirmar que, de las más de 60 mujeres entrevistadas o aludidas, la mayor parte estuvo afiliada al Partido Comunista, Partit Socialista Unificat de Catalunya o a las Juventudes de los mismos. Algunas militaban en Unió de Dones de Catalunya o en las JSU - Juventud Socialista Unificada-, nombre que adoptó la fusión de las Juventudes Socialistas y Comunistas en abril de $1936^{35}$.

También se recogen testimonios o alusiones a resistentes que pertenecian a la FAl o CNT. No aparece expresamente nombrada la Alianza Democrática Española - la organización libertaria que aglutinaba además al PSOE, UGT, IR, ERC y otros- pero indudablemente de todas estas tendencias hubo mujeres en la resistencia. Es difícil determinarlas porque destacan únicamente las de afiliación comunista o anarquista y luego su condición de "resistente" contra el fascismo, o su adhesión y colaboración con los grupos MOI (Mano de Obra Inmigrante), FTP - francotiradores y partisanos-, FFI - fuerzas francesas del interior-, "maquis», Agrupación Guerrillera Española, etc. De Segunda Montero, conocida por Conchita o "La Pequeña", enlace del grupo Huet con el de Ponzan, se dice "Sin pertenecer a ningún partido político y sin ninguna formación ideológica, movida sólo por un espíritu combativo de lucha contra la tiranía y en defensa de la libertad tomó parte activa en las luchas callejeras de la ciudad (1936)».

35 Una joven de 16 años entonces comenta "Una cosa que me hizo mucha gracia fue que pedia el ingreso en el Partido, pero me dijeron que era demasiado joven. Es decir, que para eso me encontraban demasiado joven, y no lo era para hacer todas aquellas cosas...", LINA BOSOUE, p. 76. 
Por ello, de las mujeres entrevistadas o de las que dan testimonio en el libro se puede hacer la siguiente clasificación aproximada.

Del P.C. J.S.U. Unión de Mujeres contra la guerra y el fascismo. Mujeres Antifascistas Españolas (UNE): 19.

Sin filiación expresa pero en contacto con organizaciones de resistencia antifascistas: MOI. Jeunes Filles. Grupos de Resistentes. Agrupaciones guerrilleras (casi todas de origen comunista): 40 .

CNT: 5 .

FETE: 2.

Por supuesto es imposible generalizar esta adscripción pues nos consta por otras fuentes la gran participación de mujeres pertenecientes al movimiento libertario.

Respecto a la cuantificación ni es posible determinar el número aproximado, "no habléis de cientos sino de miles" y mucho menos la adscripción por opciones políticas. Incluso los números de refugiados y de resistentes que maneja Miguel Ángel, con los cálculos de Tuñón de Lara y otras fuentes han de ser tomados como una aproximación ${ }^{36}$.

\section{Actividades como resistentes. Campos de concentración}

Al pasar la frontera fue habitual separar los hombres de las mujeres. Las madres, las esposas e hijas fueron llevadas a distintos departamentos e internadas en lugares llamados "refugios". Variaban desde un campo de concentración - Adge, St. Etienne- a cuarteles abandonados, antiguos teatros, cuadras, iglesias, o cualquier tipo de albergue habilitado rápida y provisionalmente, incluso cárceles. En general las condiciones no eran tan penosas como las que vivieron los hombres pero lo fueron mucho. Para el comandante Oria, estos "refugios" fueron la antesala de la Resistencia femenina. Allí empezaron a establecer contactos, reuniones, listas que pasaban a los campos de concentración de los hombres (Argelès, St. Cyprien, Gurs) en busca de sus familiares y para ponerse en relación con los prisioneros. Organizaron la vida en común, atendiendo los aspectos higiénicos, a los niños, enfermas y ancianos. Se negaron rotundamente a las repatriaciones forzosas. Estos "plantes" a las autoridades francesas

36 M. Ángel, Luchando en tierras.., pp. 19-20. 
solían tener como consecuencia el traslado a los llamados campos de castigo ${ }^{37}$.

Tras los «refugios» las mujeres más afortunadas van encontrando trabajo e integrándose más o menos en la sociedad rural o urbana francesa. Pero para entonces estalló la segunda guerra mundial y la consiguiente ocupación alemana. "Ocupada Francia por los nazis un nuevo abismo se abría ante nosotros... Los antifascistas de acá, de allá... sabíamos que no sería más que una nueva batalla contra el fascismo internacional... De entre los españoles refugiados se levantó un ejército de hombres y mujeres... que sería un poderoso bastión en todos los lugares de la resistencia al nazismo" ${ }^{38}$. E igual que los hombres las mujeres y las muchachas españolas se incorporaron al combate.

Una síntesis de las actividades principales comprende al menos los siguientes apartados:

1. Agentes de enlace. Transportando partes y órdenes militares, armas, dinero y a veces tabaco y alimentos. Contacto entre los diversos grupos de guerrilleros. Cadenas de evasión. Propaganda oral. La labor de enlace requería una gran resistencia moral y física. Eran las más expuestas a ser descubiertas y torturadas. Son innumerables los casos de "caídas" que resistieron hasta la muerte sin hablar. A veces tenían que recorrer $100 \mathrm{Km}$. para trasmitir un parte o trasportar una máquina de escribir, a pie, en bicicleta, en autobús...

2. Mantenimiento de "casas de apoyo". Donde se reunían para tratar los planes militares, refugiaban o convalecian los integrantes del "maquis». Además se guardaba material —como documentación virgen, sellos falsos, salvoconductos...- e instrumentos de prensa y propaganda, armas, etc. Inciuso se imprimía la prensa clandestina.

3. Sabotaje. Hay varios testimonios de sabotaje realizado en fábricas de armas por mujeres, con toda astucia, valor y sangre fría ${ }^{39}$. Ayuda a los guerrilleros saboteadores de líneas de alta tensión, ferrocarril, puentes. Todo aquello que pudiera entorpecer la actividad militar de los ocupantes.

37. La bibliogratia sobre estos primeros momentos es abundante. MARIE-CLAUDE RAFANEAU-BOJ, Les camps de concentration français en 1939. Etude sur les réfugiés espagnols en 1939. Maitrise, Université de Paris VII, 1979. Publicado con el título Odyssée pour la liberté. Les camps de prisionniers espagnols, 1939-1945, Mesnil-sur-l'Estrée, Ed. Denoël, 1993. Plages d'exil. Les camps de réfugiés espagnols en France, 1939. BDIC. Hispanistica XX, $n^{\circ}$ especial, Paris, 1989. G. DreYfuSArmand, E. Temime, Les camps sur la plague, un exil espagnol, Ed., Autrement, Paris, 1995.

38 N. Catala, De la resistencia... Su propio testimonio, p. 16.

39 N. CatAlA, De la resistencia... Testimonio de Soledad Alcon, p. 43. Luisa Alda, pp. 47-48. 
Josefa Bas, de 16 años, trabajó en el maquis de Dordogne, "destacamento mayoritariamente de españoles, más o menos ligado al "maquis" de la Corrèze». Eran unos 35 y su misión principal consistía en hacer saltar la vía ferroviaria de París entre Gourdon y Brive-La Gaillarde. "Me tuve que encargar de proporcionarles pistolas y cajas de fulminantes que nos procurábamos en Marsella, con la gente del hampa... Por medio de colectas entre los refugiados, podiamos pagar los altos precios que estas armas nos costaban... Pasábamos por caminos en los cuales, la forma de poner determinadas ramas, y otras señales... nos permitia llegar al primer control... las más olvidadas, las más injustamente tratadas" ${ }^{40}$.

\section{EL PROCESO DE LOS 40}

Para terminar haré referencia al primer proceso de la resistencia, Ilamado el Proceso de los 40, en el que hubo alrededor de 150 hombres, casi todos españoles y seis mujeres cuyos nombres constan aunque las fuentes no coinciden.

Para Neus Catalá, ellas eran: Paquita Velas, Luisa Caro, María González, Margarita, Anita Cascales, y Manolita Zapico. Miguel Ángel, coincidiendo en el número, nombra a María, Constancia, Pepita, Paquita, Rosita y Anita Cascales, Juan Marín nombra a María, Luisa, Paquita, Constancia Escuer "y muchos que yo conocía".

La redada respondió a la actuación de un destacamento español FTPMOI que actuaba en la zona ocupada, París, Bretaña y Suroeste. El número de acciones violentas entre enero de 1941 y la detención de los resistentes entre junio y noviembre de 1942 fue espectacular. Pero en estas dos grandes redadas organizadas por la Gestapo y la Policia francesa "casi todos los dirigentes del PCE y muchos guerrilleros fueron detenidos". Los hombres fueron encarcelados en La Santé. Las mujeres se beneficiaron de una cierta benevolencia, aunque no escaparon a las torturas. Paquita Velas «brutal y largamente torturada, supo mantenerse firme sin pronunciar ni una palabra ni un nombre». Maria González cuenta ella misma «me cogió de los pelos el guardia subido en una silla y levantán-

40 N. Catal.', De la resistencia... Josefa Bas, p. 66. Otras actuaciones de mujeres relata $E$. Pons, Republicanos españoles..., pp. 109, 133. Aquí alude a la liberación de Pau (agosto 1946) donde se concedieron Medallas a varios hombres y dos mujeres enlaces, Carmen Blasco y a título póstumo, Emiliana Quitian, pág. 146. En p. 296 recuerda a Pilar Ponzan Vidal, hermana del guerrillero, ambos artífices de una importante cadena de evasión. 
dome en el aire cogida de los pelos, diéronme un empujón y me lanzaron a unos metros de distancia (Luego tuve muchos sufrimientos a causa de esto)...".

La Gestapo creía que habia destrozado el movimiento. "No tenian bastante imaginación para hacerse una idea exacta de la amplitud de la organización española y de la obstinación de sus militantes" "41.

Conclusión: Para terminar adjunto uno de los escasos textos que aparecen en las publicaciones periódicas consultadas. Su título es Femmes dans la guerre, y no aparece firma ${ }^{42}$.

Depuis les agressions du fascisme dans le monde, les peuples entiers se sont insurgés contre lui; aux côtés des hommes, les femmes se sont levées. Ruseés et audacieuses, elles ont excellé dans la lutte partisane. Dernier acte d'emancipation, elles ont pris la mitrailleuse ou la grenade, et elles se sont battues.

On les a vues sur tous les fronts, dans toutes les batailles. On les a vues, menottes aux mains, dans toutes les prisons. Maltraitées autant que leurs compagnons elles se son parfois mieux tues. Elles étaient irréductibles. Sur les routes de l'exil, elles ont chanté. Sous l'uniforme rayé, elles ont succombé sans se soumettre. De tous les pays, de toutes les races, elles ont fait bloc contre l'ennemi commun.

Celles-ci sont les majuscules de la page d'histoire féminine. Lettres humbles et noires, c'est à vous que je veux rendre hommage surtout. Seules à la maison, sans mari ni argent, l'héroïsme quotidien, qu'elles ont déploye a parfois été plus méritoire. A Madrid, Anvers ou Rouen, sous les bombes et les obus, il est resté des foyers, des enfants, du bonheur. Les femmes ne se sont jamais laisses déraciner. Sans tissus, elles ont fait les vêtements. Sans chaussures, les leurs n'ont pas été pieds nus. Sans ravitaillement, elles ont nourri leur famille. Les heures de guerre, les nuits blanches, le travail plus dur, elles ont tout accepté.

Jamais abattues, elles ont relevé la tête quand même, et rendue possible la lutte du pays. A travers tant de misères, elles ont conservé l'enfance, la race de demain. Les grand'mères ont relayé les mamams disparues, les grandes soeurs ont élevé les petits frères. Elles ont préservé la chaine d'amour et maintenant, sitôt la guerre finie, elles repartent aussi fortes, aussi generouses, vers la vie et le bonheur.

Mais elles ne doivent pas oublier leurs soeurs d'Espagne.

Les premières sur la route du malheur, elles se sont pas encore rentrées. La lutte n'est pas finie...

41 M. ÁNGEL, Luchando en tierras..., p. 57.

42 L'Espagne Republicaine, $n^{0} 1,30-V I-1945$, p. 4. 
APÉNDICE: Fragmentos del testimonio escrito de Juan Marín García, Valencia, abril de $1991^{43}$.

Afiliado a las JSU. Dirigente de la FUE en la APE del Instituto Luis Vives de Valencia. Promoción bachilleres 1931-36. Voluntario a los 16 años en el batallón de la FUE de Valencia. Frente de Madrid. Reclamado por menor de edad a los cinco meses del voluntariado. Participó temporalmente en la Secretaría Técnica de la UFEH con Manuel Tuñón de Lara, Isabel Picazo, Luis Galán y otros, siendo comisario general Ricardo Muñoz Suay. A los 17 años teniente de ingenieros-trasmisiones. Frente de Teruel. Frente de Cataluña. Paso de la frontera en 1939.

En Francia internado en el campo de Vernet d'Ariège, posteriormente en el campo de concentración de Septfonds... sin pensarlo mucho da el paso decisivo: París...

A mediados de diciembre (1940), Soria, Escuer y yo nos desplazamos a... Frankfurt s/Main y no tuvimos oportunidad de volver hasta noviembre de 1941. Durante este amplio período recibimos propaganda del manifiesto de Unión Nacional, que se distribuía entre los pocos españoles que trabajábamos en la mayor industria de guerra de la zona (25.000 personas).

Llegué a Paris a mediados de noviembre de 1941... me preocupé en conseguir trabajo para los tres en el hospital psiquiátrico de Santa Ana como enfermeros... al intercambiar con nuestros compañeros nos enteramos que la organización clandestina española había trabajado con entusiasmo en este año de nuestra ausencia... a principios de 1942 me incorporo a la actividad de propaganda, consistente en la distribución de paquetes con centenares de octavillas, que eran válidas tanto para los españoles como para los franceses. En este momento y con la nueva política de la UNE propugnada por el PCE, con el beneplácito del presidente Negrín desde Londres, se abria las bases para constituir un conglomerado de todas las fuerzas sindicales y políticas... la primavera de 1942 tuvo una importancia vital al absorber como simpatizantes en la lucha a refugiados españoles sin una mentalidad política definida y a muchos miembros de familias españolas que vivian desde hacía ya años en Francia.

43 J. Marin García, "Los exiliados españoles durante el periodo febrero 1939-1945. Testimonio de mi participación en el "Proceso de los Cuarenta" ". Cuadernos Republicanos, $n^{2} 23$ (julio 1995), pp. 113-140. 
En el domicilio de Soria y Paquita Velas se había ubicado la estafeta central de propaganda. Soria era persona muy bromista, confiado y que se acoplaba fácilmente a los riesgos sin darle mayor importancia... la afluencia de colaboradores, el carácter tertuliano de Juan y Paquita, que yo les reprobaba por falta de previsión, originó el aislarme prudentemente de este domicilio... En el momento de los acontecimientos que van a proceder, en el mismo hotel que vivia yo, habia una familia española compuesta por María, su compañero Juan Núñez y una niña de unos tres años.

\section{El proceso de los 40}

En la madrugada del veintisiete de junio de 1942 la policía francesa llamó a mi puerta diciéndome que traían una orden de detención de María y Juan pero como el conserje les había dicho que yo también era español me rogaban les acompañase a la Jefatura de Policía... Ya se encontraban Juan, Paquita, Caro, su compañera, Vizcaino, Calpe, Escuer y muchos que yo conocía... La primera impresión fue que el golpe sólo afectaba a la ciudad de París, pero en las dos o tres horas que permanecí en la sala, iban llegando más compatriotas detenidos y supuse que alguno de ellos y por no conocerlos serian de provincias, aunque yo no vi ningún enlace conocido...

Mi situación se complicó a raíz de un nuevo interrogatorio... centró sus preguntas sobre mi estancia en el hospital psiquiátrico... a los pocos días vinieron a por mi miembros de la Gestapo para trasladarme a la Jefatura de la misma calle Saussais, junto con otros detenidos de ambos sexos de distintas nacionalidades... María me previno manifestando que al interrogarle sobre mi le habían dicho claramente que por ser el único al que no habian podido implicarme en nada me consideraban como uno de los jefes de la red. Pude comprobar que las torturas y malos tratos habian afectado a un núcleo muy reducido, destacando Paquita Velas, María González, Juan Soria, Guillermo Pires, Constanza...

Un grupo de cinco españoles colocamos enfrentados dos pequeños bancos y nos sentamos para iniciar un diálogo que duraría hasta el amanecer... un análisis de la situación muy grave pues en contra de las noticias recibidas del exterior nosotros y a la vista del número de detenidos del orden de 200 no podiamos aceptar... la interpretación condescendiente de la dirección... se efectuó un análisis sobre las medidas a tomar para que no se perdiese la cohesión entre todos nosotros y las mujeres, ya que conocíamos que tanto en la prisión de La Santé, donde iriamos los hombres como en la de La Roquette, para las mujeres, los detenidos políticos y comunes estaban juntos. 
Sobre el 14 de octubre de 1943 el juez de instrucción y por oficio concedió el "non lieu»... a la mayoría de los encartados en nuestro affaire. Se comentó que los jueces fueron sensibles a los toques de advertencia de radio Londres... Las condenas se consideraron benignas en todos los casos, ya que las más graves fueron de dos años.

Mis compañeros me aconsejaron el regreso a España y así lo hice. Estuve en tránsito por la cárcel de Burgos, Porlier, Albacete, La Modelo de Valencia y la cárcel de Castellón... Tras un juicio informal, mi lesión no me sirvió de mucho, pues me enviaron castigado a hacer el servicio militar en África. 